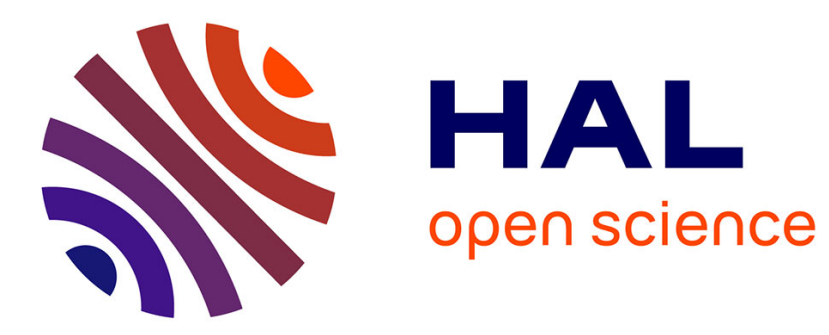

\title{
Extension du modèle de Freedman de formation des échos au cas transitoire
}

\author{
R. Raillon, A. Lhémery, D. de Vadder
}

\section{To cite this version:}

R. Raillon, A. Lhémery, D. de Vadder. Extension du modèle de Freedman de formation des échos au cas transitoire. Journal de Physique IV Proceedings, 1994, 04 (C5), pp.C5-829-C5-832. 10.1051/jp4:19945179 . jpa-00252860

\section{HAL Id: jpa-00252860 https://hal.science/jpa-00252860}

Submitted on 1 Jan 1994

HAL is a multi-disciplinary open access archive for the deposit and dissemination of scientific research documents, whether they are published or not. The documents may come from teaching and research institutions in France or abroad, or from public or private research centers.
L'archive ouverte pluridisciplinaire HAL, est destinée au dépôt et à la diffusion de documents scientifiques de niveau recherche, publiés ou non, émanant des établissements d'enseignement et de recherche français ou étrangers, des laboratoires publics ou privés. 


\title{
Extension du modèle de Freedman de formation des échos au cas transitoire
}

\author{
R. RAILLON, A. LHÉMERY et D. DE VADDER
}

Ecole Centrale Paris, Laboratoire de Mécanique, URA 850 du CNRS, Equipe Ultrasons, Grande Voie des Vignes, 92295 Châtenay-Malabry cedex, France

\begin{abstract}
This paper presents an extension of a model presented by the same authors in the previous CFA [1]. The model allows fast computations of the acoustical impulse-response of the problem of the radiation of ultrasonic pulses by realistic transducers, their scattering by immersed targets and their reception. In the previous version, the model was restricted to the case of small targets centered on the transducer axis. Here, it is shown that a more general formula can be derived allowing the computation of the echo-responses from targets not necessarely centered on axis which can be of arbitrary size. It is shown that the present model may be considered as an extension to the transient case of the well-known Freedman's model which derived from a CW theory. Basically, the idea is to restrict the computation to the contributions arising from the only parts of the target where interferences are constructive.
\end{abstract}

\section{INTRODUCTION.}

Lors du précédent CFA, nous avions présenté un modèle géométrique permettant de calculer les échos renvoyés par des cibles immergées de formes simples, centrées sur l'axe du traducteur, et insonifiées par des traducteurs pistons, disques plans [1]. Ce modèle a été étendu à d'autres cas de géométries [2]. Nous allons présenter le résultat le plus général obtenu et comparer ce nouveau modèle à celui de Freedman dont il peut apparaître comme une extension au cas de l'imagerie large bande.

\section{PRÉSENTATION DU MODÈLE DE FREEDMAN [3].}

Freedman se place dans le cadre de l'acoustique linéaire et de l'approximation de Kirchhoff (les phénomènes de diffraction multiple et les ondes rampantes ne sont pas pris en compte). La cible est rigide et immergée dans un fluide idéal. Freedman considère un émetteur et un récepteur ponctuels auxquels il superpose une directivité pour prendre en compte approximativement les effets de diffraction à l'émission et à la réception. Le signal transmis est une impulsion à amplitude modulable avec une enveloppe quelconque et dont le spectre est "à bande étroite". Dans la plupart des cas traités par Freedman, la distance cible-traducteur est grande et le front d'onde incident peut être supposé plan sur l'étendue de la cible. L'émission et la réception sont toujours séparées dans le temps.

L'équation de départ de Freedman exprimant l'écho $E$ renvoyé par une cible de surface insonifiée $S$ et mesuré par le récepteur s'écrit

$$
E=-\frac{i}{\lambda} \exp (i \omega t) \iint_{S} \frac{D_{p}(\mathbf{r}, \theta) D_{H}(\mathbf{r}, \theta) \cos \psi}{r^{2}} \exp (-2 \mathbf{i} \mathbf{k} . \mathbf{r}) d S,
$$

où $D_{p}$ et $D_{h}$ sont les directivités du traducteur pour l'émission et la réception, $(\mathbf{r}, \theta)$ repère la position d'un point courant de la surface $S$ de la source, $\mathbf{k}$ est le vecteur d'onde, $\omega$ la pulsation, $\lambda$ la longueur d'onde, et $\psi$ l'angle que fait l'onde plane incidente avec la normale à la surface $d S$ en $(\mathbf{r}, \theta)$.

Freedman calcule un développement asymptotique de l'équation (1) et observe que l'écho renvoyé par une cible immergée est en fait composé de plusieurs échos générés en des zones particulières de la surface de la cible, celles présentant une discontinuité, selon la distance $\mathbf{r}$, dans la fonction $A_{\mathfrak{y}}(r)$ ou dans 
une de ses dérivées $d^{n} A_{w}(r) / d r^{n} . A_{w}(r)$ est la surface projetée de la cible en fonction de la distance $r$, pondérée par les fonctions de directivités du traducteur.

Ainsi, l'écho reçu par le récepteur est donné par,

$$
\begin{gathered}
E=\sum_{g=1}^{f} E_{g}, \\
E_{g}=\frac{i}{\lambda r_{m}^{2}} \exp \left[i\left(\omega t-2 k r_{1}\right)\right] \exp \left[-2 i k\left(r_{g}-r_{1}\right)\right] \sum_{n=0}^{\infty} \frac{D\left(A_{w}, g, n\right)}{(i 2 k)^{n}} .
\end{gathered}
$$

$D\left(A_{w}, g, n\right)$ est la discontinuité de $d^{n} A_{w}(r) / d r^{n}$ à la distance $r_{g}, r_{1}$ est la plus courte distance entre la cible et le traducteur. L'expression (3) est obtenue pour des distances cibles traducteur grandes permettant l'approximation $1 / r^{2} \approx 1 / r_{m}{ }^{2}$ avec $r_{m}$ distance moyenne de la cible. L'enveloppe de chaque écho $E_{g}$, que nous appellerons "écho élémentaire", a la même forme que celle du signal incident.

\section{PRÉSENTATION DU MODÈLE TRANSITOIRE SIMPLIFIÉ}

Nous nous plaçons dans l'hypothèse d'une source de vitesse particulaire $v(\mathbf{r}, t)$ à variables espace et temps séparées $v(\mathbf{r}, t)=v(t) \Gamma(\mathbf{r})$ (hypothèse de fonctionnement en piston). L'écho $O(t)$ reçu par le traducteur après diffraction par une cible immergée s'écrit

$$
O(t)=I_{r e f}(t) * H(t)
$$

$H(t)$ est la réponse impulsionnelle acoustique prenant en compte tous les phénomènes de diffraction intervenant au niveau du traducteur, de la cible et lors de la propagation et $I_{r e f}(t)$ le signal de référence prenant en compte les transductions éléctro-acoustique, acousto-électrique et la forme de l'excitation électrique. $I_{r e f}(t)$ est obtenu simplement en mesurant, par exemple, l'écho renvoyé par un plan infini placé très près de la surface du traducteur [2].

On garde essentiellement les mêmes hypothèses que Freedman, sauf que l'on prend en compte une excitation temporelle quelconque et que les effets de diffraction par le traducteur sont pris en compte exactement, à l'émission comme à la réception. La réponse impulsionnelle acoustique $H(t)$ s'écrit [4],

$$
H(t)=\iint_{S} \frac{\cos \theta}{\rho_{0} c} h^{\partial t}\left(\mathbf{r}_{S}, t\right) * h^{\partial t}\left(\mathbf{r}_{S}, t\right) d S_{S} .
$$

$t$ est le temps, $\mathbf{r}_{S}$ repère un point courant à la surface du traducteur et $\mathbf{r}_{C}$ le centre du traducteur. $\theta$ est l'angle formé par le vecteur $\mathbf{r}_{S^{-}} \mathbf{r}_{C}$ et la normale à $\mathbf{d} \mathbf{S}_{S}$ en $\mathbf{r}_{S}$ (voir Fig. 1 ). $\rho_{0} c$ est l'impédance spécifique du milieu. $h^{\partial t}\left(\mathbf{r}_{s}, t\right)$ est la réponse impulsionnelle pour la pression émise en $\mathbf{r}_{S}$ et est donnée par l'intégrale de Rayleigh dépendante du temps,

$$
h^{\partial t}\left(\mathbf{r}_{S}, t\right)=\rho_{0} \frac{\partial}{\partial t} \iint_{T} \Gamma\left(\mathbf{r}_{T}\right) \frac{\delta\left(t-\mid \mathbf{r}_{S}-\mathbf{r}_{T} \bigvee / c\right)}{2 \pi\left|\mathbf{r}_{S}-\mathbf{r}_{T}\right|} d S_{T},
$$

où $T$ est la surface active du traducteur et $\Gamma$ est sa répartition spatiale de source (et sa sensibilité).

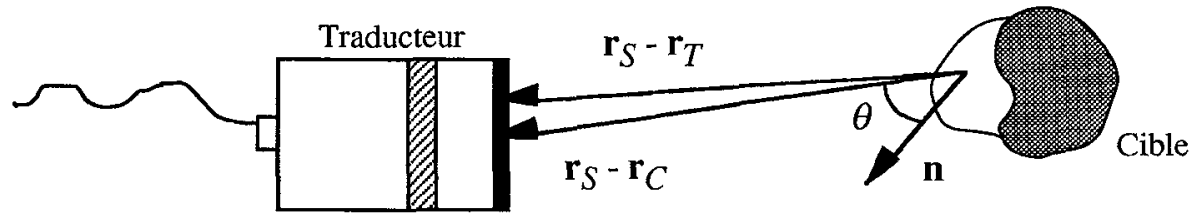

Fig. 1 - Géométrie de la situation étudiée et définition des notations principales.

Comme Freedman, on utilise le fait que seules certaines parties de la cible contribuent à un écho non nul, nous avons simplifié l'expression (5) et obtenu l'expression suivante pour l'écho renvoyé par une cible de forme simple $[1,2]$, (toujours dans le cas où elle est loin du traducteur): 


$$
O(t)=I_{r e f}(t) * \frac{1}{2 \rho_{0}} \sum_{i=1}^{n} P I R\left(\mathbf{r}_{i}, t\right) * R P(t)
$$

$P I R\left(\mathbf{r}_{i}, t\right)=h^{\partial t}\left(\mathbf{r}_{i}, t\right) * h^{\partial t}\left(\mathbf{r}_{i}, t\right)$, est la réponse impulsionnelle en émission-réception pour un point $\boldsymbol{r}_{i} \cdot R P(t)$ est la fonction connue sous le nom de profil réflecteur de la cible; elle est identique à la dérivée première de $A_{w}(r)$.

L'équation (7) exprime que l'écho global renvoyé par la cible est la somme d'échos issus de zones particulières de sa surface, centrées en $\mathbf{r}_{i}$ et où le profil réflecteur de la cible, ou une de ses dérivées, présente une discontinuité.

\section{DISCUSSION.}

Les interprétations physiques de la formation des échos, d'après les deux modèles, sont proches; on retrouve que seules certaines zones de la cible (zones utiles de diffraction) contribuent à un écho non nul. On calcule l'écho pour les zones où les interférences sont constructives et pas où elles sont destructives, d'où une importante économie de temps de calcul.

Le calcul quasi-exact (d'après Eq.(5)) des réponses impulsionnelles de cibles billes, cônes et disques a permis, grâce à l'interprétation du champ d'un traducteur piston plan en termes d'onde géométrique et d'onde de bord [5], d'interpréter géométriquement ce résultat et de calculer précisément la dimension de ces zones utiles quand elles se situent autour de l'axe du traducteur [6].

Les deux modèles sont quantitatifs. D'après Freedman, l'amplitude d'un écho élémentaire dépend de la valeur de la discontinuité de la fonction $A_{w}(r)$ et de l'ordre de dérivation auquel elle apparaît. Avec le modèle transitoire simplifié, une très bonne concordance quantitative avec l'expérience est obtenue [6].

L'avantage du modèle transitoire simplifié est de pouvoir prévoir les formes temporelles d'échos transitoires alors que le modèle de Freedman, fondé sur une théorie monochromatique, ne le peut pas.

L'interprétation physique de l'approximation qui a permis la simplification de l'expression de l'écho impulsionnel (passage de Eq.(5) à Eq.(7)) permet de bien comprendre les limites du modèle transitoire simplifié. Mais il est difficile de quantifier ces limites. Il faut garder à l'esprit que l'approximation faite consiste à considérer que les formes temporelles des contributions de tous les points d'une zone de lá cible qui génère un écho sont les mêmes, comme si tous ces points se trouvaient au centre de cette zone. La zone est d'autant plus étendue que la cible est près du traducteur et l'approximation est d'autant moins valable que la zone est étendue. Cela interdit aussi les cibles présentant une surface plane étendue perpendiculaire à l'axe du traducteur.

Un autre point intéressant du modèle transitoire simplifié est la possibilité de calculer les réponses impulsionnelles et pas seulement les échos. Cela permet d'interpréter les résultats et les mécanismes de formation des échos sans que la "physique soit cachée" par des effets de transduction électro-acoustique.

\section{RÉSULTATS DU MODÈLE SIMPLIFIÉ TRANSITOIRE.}

Le modèle simplifié permet d'obtenir très rapidement des échos comparables avec l'expérience.

Il faut, dans le cas d'un traducteur à profil d'amplitude vibratoire uniforme (le cas des traducteurs non uniformes est plus complexe [6]):

- mesurer le signal de référence $I_{r e f}(t)$, c'est à dire enregistrer l'écho renvoyé par un plan infini placé très près du traducteur et perpendiculairement à son axe (il existe d'autres moyens d'obtenir $I_{r e f}(t)$ [6] ou [7]). - calculer le profil réflecteur $R P(t)$ de la cible et les fonctions $P I R\left(\mathbf{r}_{i}, t\right)$ qui interviennent aux différents points de discontinuité du profil réflecteur de la cible, et les convoluer. Dans le cas d'un traducteur disque plan, $h^{\partial t}\left(\mathbf{r}_{i}, t\right)$ est connu analytiquement et des programmes simples font ces calculs en quelques secondes, - sommer les différents échos ainsi obtenus et correctement situés dans le temps. On a alors la réponse impulsionnelle en émission réception.

- convoluer le résultat de la somme avec le signal de référence $I_{\text {ref }}(t)$ pour obtenir l'écho global.

La figure 2 illustre les différentes étapes nécessaires pour calculer la réponse impulsionnelle d'un cône centré. Les zones du cône générant des échos sont situées autour de sa pointe et de sa périphérie. Il faut donc calculer la fonction PIR deux fois, pour un point sur l'axe et pour un point de la périphérie du cône. Le résultat ainsi obtenu très rapidement est comparé avec le résultat d'un modèle transitoire non simplifié [4,7], déjà validé par ailleurs [7].

Pour les cas de la figure 3 , une seule fonction PIR est à calculer car la cible est petite (cas b)) ou parce que les discontinuités de son profil réflecteur sont situées à la même distance de l'axe et quasiment à la même distance du traducteur (cas d) des bords proche et lointain d'un disque incliné centré. .

Des comparaisons d'échos calculés avec le modèle simplifié et d'échos expérimentaux doinnent de bons résultats tant quantitatifs que qualitatifs [6]. 


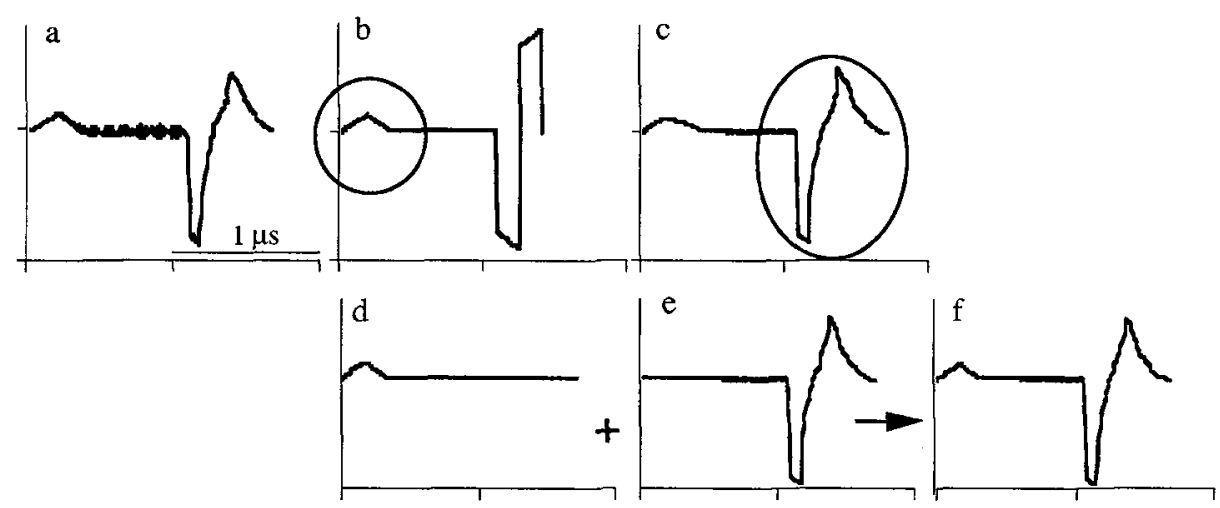

Fig. 2 Réponse impulsionnelle d'un cône de $150^{\circ}$, de rayon $r_{m}=3 \mathrm{~mm}$ à la distance $z 0=200 \mathrm{~mm}$ d'un traducteur de $\varnothing 20 \mathrm{~mm}$. -a) Calculée selon Eq. (5) (modèle non-simplifié), -b) Convolution de $R P(t)$ avec $P I R$ calculée pour un point sur l'axe à la distance $\left.z_{0},-\mathrm{c}\right)$ Convolution de $R P(t)$ avec $P I R$ calculée pour un point désaxé de $r_{m}$, à la distance $z_{0}$, -d) première partie de b), -e) dernière partie de c), -f) Somme de d) et e),à comparer avec a).
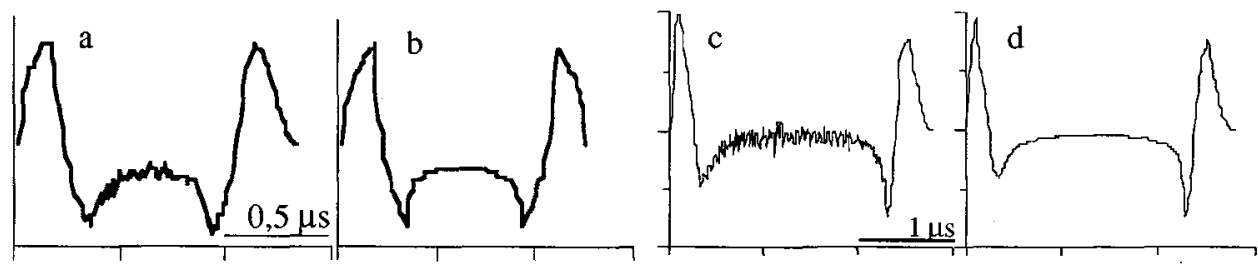

Fig. 3 a), et b): Réponses impulsionnelles d'un disque $\varnothing 2 \mathrm{~mm}$, incliné de $20^{\circ}$, à la distance $z_{0}=120 \mathrm{~m}$ d'un traducteur $\varnothing 20 \mathrm{~mm}$. -a) Calculée avec le modèle transitoire non simplifié, -b) Convolution de $R P(t)$ avec $P I R$ calculée pour un point sur l'axe, à la distance $z_{0} . c$ ) et d): Même chose mais disque de $\emptyset=2 a$ $=10 \mathrm{~mm}$, incliné de $20^{\circ}$, à la distance $z_{0}=200 \mathrm{~mm}$. -c) calculée avec le modèle transitoire non simplifié, -d) Convolution de $R P(t)$ avec $P I R$ calculée pour un point désaxé de " $a \cos 20^{\circ}$ ", à la distance $z 0$.

\section{CONCLUSION.}

Nous avons présenté un modèle qui permet un calcul rapide d'échos et une interprétation physique du mécanisme de formation de ces échos. Ce modèle ne fonctionne pas dans tous les cas de figures. Par exemple il ne fonctionne pas pour des cibles dans le champ très proche du traducteur ou pour des cible dont une grande partie de la surface est plane et orientée perpendiculairement à l'axe du traducteur.

Ce modèle simplifié a été utilisé dans différentes applications du contrôle non destructif, où de nombreux paramètres interviennent. Par exemple, nous avons étudié l'effet de la géométrie du réflecteur utilisé lors de la caractérisation des traducteurs en émission-réception ou le problème de la discrimination des défauts [6].

Dans l'avenir, nous voulons utiliser ce modèle pour étudier les échos renvoyés par des cibles insonifiées par des traducteurs plans à profil non uniformes [8]. Nous espérons aussi étendre le modèle au cas de la propagation des ondes élastiques.

[1] Raillon R., Lhémery A. et De Vadder D., "Détermination géométrique des réponses impulsionnelles échographiques sur des cibles immergées de forme simple", 2 ème Congrès Français d'Acoustique Volume II, J. Roux Ed., Arcachon France avril 1992 (Les Éditions de Physique, Les Ulis, 1992) pp. 831-834

[2] Raillon R., Lhémery A. et De Vadder D., to be published in Review of Progress in QNDE, Vol. 13-A, eds.D.O. Thompson and D.E. Chimenti (Plenum, New York, 1994).

[3] Freedman A., Acustica 12 (1962) 61-70.

[4] Lhémery A., J. Acoust. Soc. Am. 90 (1991) 2799-2807.

[5] Weight J.P. and Hayman A.J., J. Acoust. Soc. Am.. 63 (1978) 396-404.

[6] Raillon R., PhD dissertation, École Centrale Paris (in french), (1993).

[7] Lhémery A. and Raillon R., to be published in J. Acoust. Soc. Am.

[8] Lhémery A., De Vadder D. et Dimitriu S., these proceedings. 